\title{
Environmental and biological influences on the stability of silver nanoparticles
}

\author{
LIU Wei, ZHOU QunFang, LIU JiYan, FU JianJie, LIU SiJin \& JIANG GuiBin* \\ State Key Laboratory of Environmental Chemistry and Ecotoxicology, Research Center for Eco-environmental Sciences, Chinese Academy of \\ Sciences, Beijing 100085, China
}

Received November 10, 2010; accepted December 14, 2010

\begin{abstract}
Silver nanoparticles (Ag NPs) are easily dispersed and stable, and their application has become widespread. Consequently, concern has arisen regarding their potential effects on the environment and human health. However, the current understanding on the stability of Ag NPs is limited. Therefore, in this study the influence of environmental and biological factors, such as ionic strength, valence, biological molecules, $\mathrm{pH}$ and temperature, on the properties of Ag NPs was investigated. The absorption spectra, size, zeta potential, and release of silver ions were evaluated. Ionic strength, valence, Dulbecco's Modified Eagle's Medium (DMEM), without fetal bovine serum (FBS), reduced the stability of the Ag NPs. However, in DMEM with 10\% FBS, the Ag NPs maintained their stability and nanoscale characteristics. These findings provide an essential insight into the environmental and biological factors that should be evaluated in a risk assessment of Ag NPs.
\end{abstract}

silver nanoparticles, environmental influence, biological influence, stability

Citation: $\quad$ Liu W, Zhou Q F, Liu J Y, et al. Environmental and biological influences on the stability of silver nanoparticles. Chinese Sci Bull, 2011, 56: 2009-2015, doi: 10.1007/s11434-010-4332-8

Nanomaterials have at least one dimension that is $1-100$ nm. On this scale, materials have unique physical and chemical properties such as high specific surface area, tunable fluorescence and electron tunneling effect [1-3]. Because of these properties, nanomaterials have potential applications in optical, electronic and medical products $[4,5]$. Among the different nanomaterials, silver nanoparticles (Ag NPs) are one of the fastest growings. They are used as antibacterial agents, catalysts, medicines, biological sensors and more [6-11]. Ag NPs could potentially be released into the environment from these products, and consequently pose a potential toxicity risk to human health $[12,13]$. However, little is currently known about the likely adverse effects of Ag NPs on health.

The biological activity of Ag NPs depends on many features, such as their size [14], surface coatings [15] and release of silver ions [16]. Their size and the release of silver

\footnotetext{
*Corresponding author (email: gbjiang@ rcees.ac.cn)
}

ions are regulated by the Ag NPs stability [17]. A reduction in their stability can result in aggregation of the Ag NPs, which consequently leads to the total or partial lose of their nanoscale properties. The aggregation of Ag NPs also alters their cellular uptake and the response of cells to the NPs [18], and modifies their bioavailability and toxicity [19]. The status of Ag NP aggregation or dispersion determines the magnitude of their toxicity and the mechanism of their environmental and biological effects. Although NP clusters have been observed in some toxicological studies, few of these studies have considered the aggregation kinetics of $\mathrm{Ag}$ NPs [20]. Aggregation may change the NP characteristics and lead to different conclusions. Therefore, it is crucial to evaluate the status of Ag NPs in environmental and biological medium prior to determining their environmental risk and toxicity.

In this study, the influence of environmental and biological factors on the properties of Ag NPs, such as absorption peak, size, zeta potential and ion release, was 
investigated. The results of this study are essential for toxicity assessment of Ag NPs.

\section{Materials and methods}

\subsection{Chemicals and reagents}

Ag NPs were purchased from Huzheng Nano Technology Limited Company (Shanghai, China). The particles were purified by centrifugation three times at $12000 \mathrm{r} / \mathrm{min}$ for $20 \mathrm{~min}$. The supernatant was discarded and the solid was resuspended in deionized (DI) water and characterized by UV-Vis spectrometry (Beckman Coulter DU 800, USA), nano particle size analysis (N5, Backman, USA), inductively coupled plasma mass spectrometry (ICP-MS, Agilent 7500, USA) and transmission electron microscopy (TEM, Hitachi H-7500, Japan). The stock suspension was stored at $4^{\circ} \mathrm{C}$ in the dark.

Salt reagents $\left(\mathrm{NaCl}, \mathrm{CaCl}_{2}, \mathrm{MgCl}_{2}, \mathrm{FeCl}_{3}\right.$, ) were AR grade and purchased from the Beijing Chemical Reagent Company (Beijing, China). High Glucose Dulbecco's Modified Eagle's Medium (DMEM) and fetal bovine serum (FBS) were purchased from HyClone and Invitrogen (USA), respectively.

\subsection{Preparation of Ag NPs in different systems}

Suspensions of $100 \mu \mathrm{mol} / \mathrm{L} \mathrm{Ag} \mathrm{NPs} \mathrm{were} \mathrm{obtained} \mathrm{by} \mathrm{di-}$ luting the stock solution of $\mathrm{Ag}$ NPs with either $\mathrm{NaCl}, \mathrm{CaCl}_{2}$, $\mathrm{MgCl}_{2}$ or $\mathrm{FeCl}_{3}$ at concentrations of $100 \mu \mathrm{mol} / \mathrm{L}, 1 \mathrm{mmol} / \mathrm{L}$, $10 \mathrm{mmol} / \mathrm{L}$, and $0,0.1,0.2,0.3,0.4,0.5,0.6,0.7,0.8,0.9$, and $1.0 \mathrm{~mol} / \mathrm{L}$. The absorption at $400 \mathrm{~nm}$ was measured 30 min and $8 \mathrm{~h}$ later by UV-Vis spectrometry.

The $\mathrm{pH}$ values of the $100 \mu \mathrm{mol} / \mathrm{L}$ Ag NP suspensions were adjusted with $1 \mathrm{~mol} / \mathrm{L} \mathrm{HCl}$ and $1 \mathrm{~mol} / \mathrm{L} \mathrm{NaOH}$ to 4.2 , 5.4, 6.4, 7.6 and 8.4. After $30 \mathrm{~min}$ and $8 \mathrm{~h}$, the absorption at $400 \mathrm{~nm}$ was measured by UV-Vis spectrometry.

The $100 \mu \mathrm{mol} / \mathrm{L} \mathrm{Ag}$ NPs suspensions were also prepared in FBS-free DMEM and DMEM with 10\% FBS (DMEM+ FBS). The absorption at $400 \mathrm{~nm}$ was measured $30 \mathrm{~min}$ and $8 \mathrm{~h}$ later by UV-Vis spectrometry.

These Ag NP suspensions were stored at 4,20 and $37^{\circ} \mathrm{C}$ for $8 \mathrm{~h}$. After storage the adsorption at $400 \mathrm{~nm}$ was measured by UV-Vis spectrometry.

\subsection{Particle size analysis and zeta potential measurements}

Particle size and zeta potential of Ag NPs in suspensions of $0.2 \mathrm{~mol} / \mathrm{L} \mathrm{NaCl}, \mathrm{CaCl}_{2}$, DMEM and DMEM+FBS were measured by laser particle size analyzer (Malvern Nano ZS, Malvern, UK). All experiments were carried out below $20^{\circ} \mathrm{C}$.

\subsection{Assessment of released silver ions}

The free silver ions in various Ag NP suspensions were measured using a Silver Ion-Selective Electrode (Ag-ISE, Jenco, Shanghai, China). A calibration curve was constructed using the diluted $\mathrm{AgNO}_{3}$ solution. The $\mathrm{Ag}^{+}$concentration was calculated from the obtained data using the linear calibration.

\subsection{Statistics}

All statistical analyses were performed using Sigma Plot 10.0 (Systat Software Inc., San Jose, CA). All results are presented as the mean $\pm \mathrm{SD}$ of at least three independent experiments. The statistical significance of the experimental data was analyzed by Student's $t$-test, and a $P$ value of $<0.05$ determined statistical significance.

\section{Results and discussion}

\subsection{Characterization of Ag NPs}

TEM imaging showed that the Ag NPs were homogeneously dispersed spheres (Figure 1). The concentration of the stock solution was $4.89 \mathrm{mmol} / \mathrm{L}$ and the particle size was $23.8 \pm 6.7 \mathrm{~nm}$. An absorption peak typical of Ag NPs was observed at $400 \mathrm{~nm}$.

\subsection{Influence of ionic strength and valence on stability of the Ag NPs}

The absorption peak at $400 \mathrm{~nm}$ confirmed that Ag NPs were present in the solution. Consequently, any decrease in the absorption and/or increase in the full width at half maximum (FWHM) could be taken as an indication that the $\mathrm{Ag}$ NPs had aggregated and formed bigger particles. Disappearance of the $400 \mathrm{~nm}$ peak suggests that the Ag NPs have lost their stability and the particle size has increased to microscale [21]. When the particles were in suspension with $0.5 \mathrm{~mol} / \mathrm{L} \mathrm{NaCl}$, which has an ionic strength of $0.5 \mathrm{~mol} / \mathrm{L}$, the absorption at $400 \mathrm{~nm}$ decreased slightly and the FWHM did not change (Figure 2 (a) and (b)). By contrast, in 0.5 $\mathrm{mol} / \mathrm{L} \mathrm{CaCl}_{2}$, which has an ionic strength of $2 \mathrm{~mol} / \mathrm{L}$, the

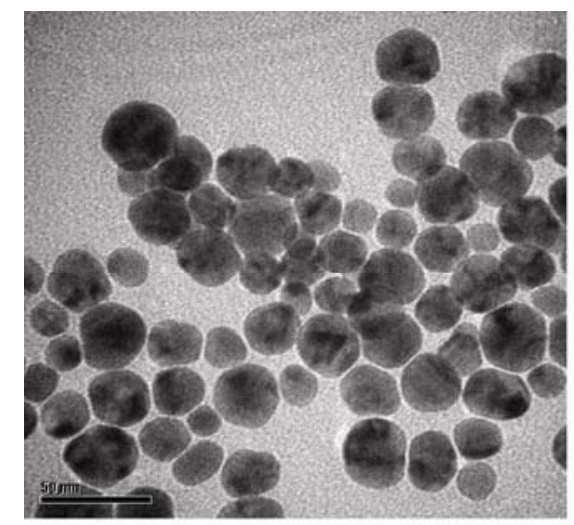

Figure 1 TEM image of Ag NPs. Magnification: $\times 80000$. 
absorption at $400 \mathrm{~nm}$ decreased from 1.8 to 0.5 , and the FWHM increased significantly (Figure 2(c) and (d)). This finding suggests that increased ionic strength can induce aggregation of the Ag NPs and loss of the stable dispersion. Additionally, it indicates that the higher valence readily induces the loss of the stable dispersion of the Ag NPs. Results from the $\mathrm{Mg}^{2+}$ suspensions confirmed the effect of cation valence. In addition to the decrease in the absorption at $400 \mathrm{~nm}$, another peak at $500 \mathrm{~nm}$ emerged in the $\mathrm{Mg}^{2+}$ suspensions, which is indicative of the formation of larger particles (Figure 3). Iron had a pronounced effect on the stability of the Ag NPs, and $200 \mu \mathrm{mol} / \mathrm{L} \mathrm{Fe}^{3+}$ solution dissolved all the particles (Figure 4). This might have occurred because of the high cation valence and the oxidative properties of the $\mathrm{Fe}^{3+}$ ions.

The salt solutions used in this study are common in natural aqueous environmental and biological solutions. These findings indicate that Ag NPs will tend to aggregate in high ionic strength and valence solutions, and lose their nanoscale properties. This observation is consistent with an earlier study on nano- $\mathrm{TiO}_{2}[22]$.

\subsection{Influence of biological molecules on the stability of the Ag NPs}

For the in vitro experiments, the nanomaterials were suspended in cell culture medium prior to exposure to cells or bacteria. The influence of the cell culture medium on
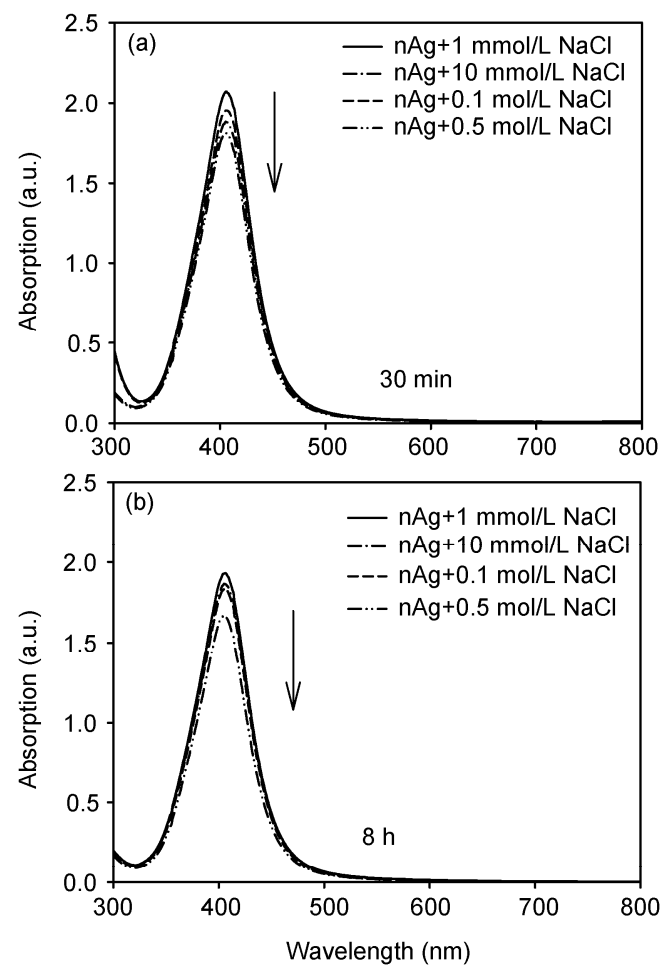

stability of Ag NPs was evaluated.

After $8 \mathrm{~h}$ of exposure to FBS free-DMEM, which is a common cell culture medium, the specific absorption peak of the Ag NPs disappeared and a peak for the aggregates appeared at $680 \mathrm{~nm}$ (Figure 5). This indicates that the components in DMEM (salt and amino acid) led to aggregation of the Ag NPs. However, the addition of $10 \%$ FBS kept the Ag NPs in a well-dispersed state, which suggests that FBS could protect the Ag NPs from aggregating. This protective effect was also observed in another common culture medium, RPMI1640 [23]. The protective role of FBS might occur because Ag NPs bind to proteins in the FBS. As a result, the surface of the Ag NPs would be covered in FBS, which could then prevent aggregation [24]. Thus, Ag NPs would remain dispersed in any medium containing FBS. Ag NPs would probably maintain their nanoscale properties under in vivo conditions as there are abundant proteins in blood and biological liquids that could act in a similar manner.

\subsection{Effect of temperature on the stability of the Ag NPs}

Temperature is one of the important environmental factors that influence the stability, activity and chemical characteristics of materials. The effect of temperature on the stability of the Ag NPs was investigated at 4,20 and $37^{\circ} \mathrm{C}$. The $\mathrm{Ag}$ NPs in DI water remained intact at all temperatures tested (Figure 6). In the salt solutions and DMEM, the
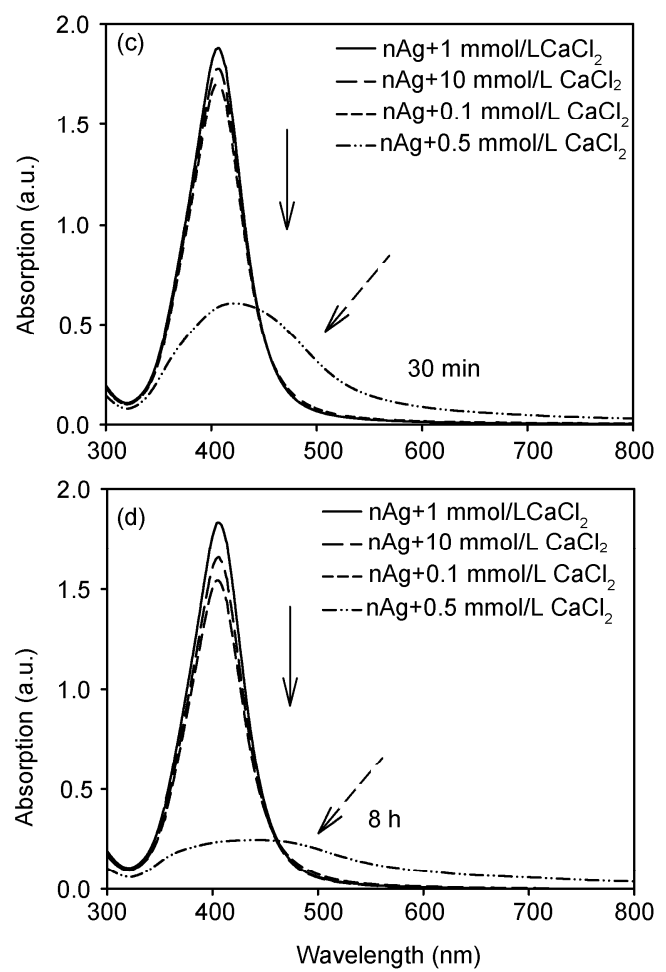

Figure 2 The UV-Vis adsorption spectra of $\mathrm{Ag} \mathrm{NPs}$ diluted in $\mathrm{NaCl}$ and $\mathrm{CaCl}_{2}$ solutions. Solid arrows indicate the typical absorption peak of Ag NPs, and the dotted arrows indicated where the absorption is decreasing and the FWHM is increasing. 


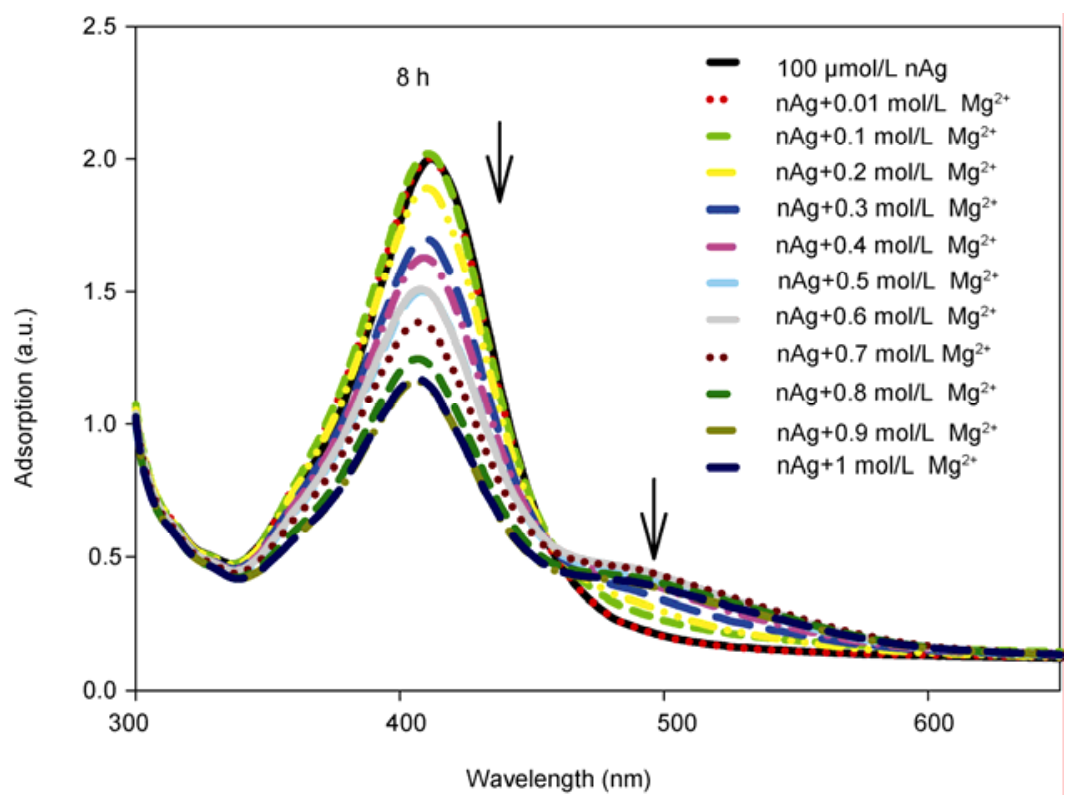

Figure 3 The UV-Vis adsorption spectra of Ag NPs diluted in $\mathrm{MgCl}_{2}$ solution. Arrows show the typical absorption peak of Ag NPs and the peak of larger particles at $500 \mathrm{~nm}$.

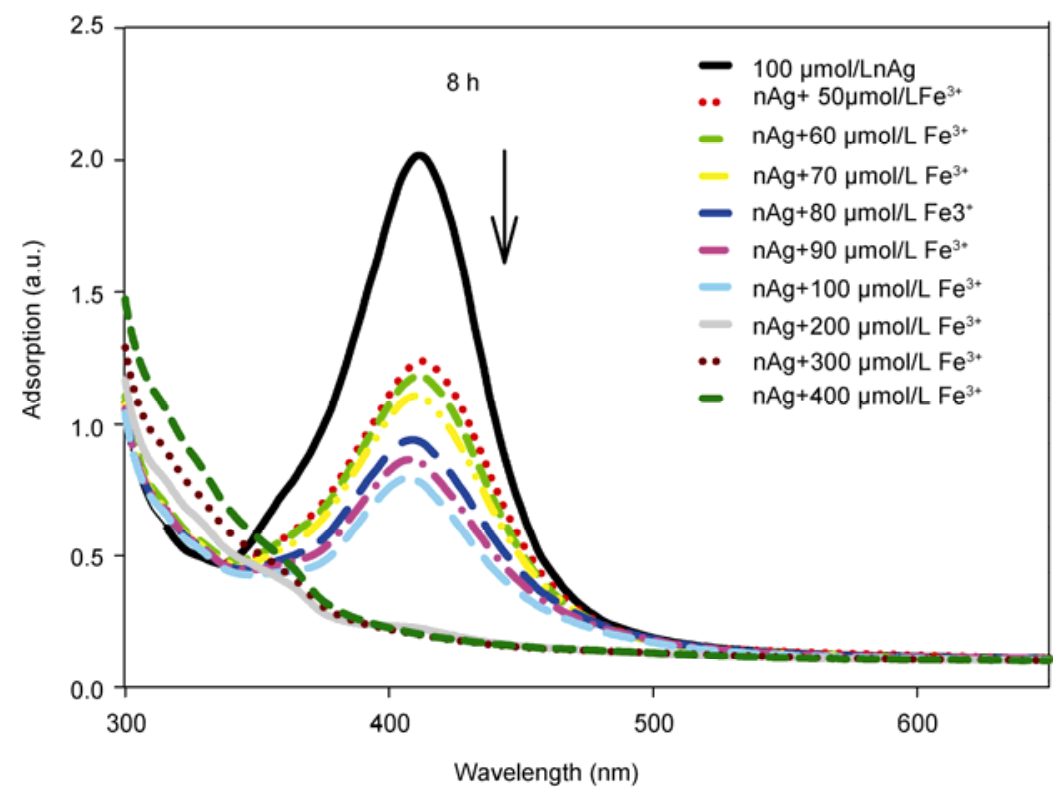

Figure 4 The UV-Vis adsorption spectra of Ag NPs in the $\mathrm{FeCl}_{3}$ solution. The arrow indicates the decrease in the absorption at $400 \mathrm{~nm}$.

increase in temperature facilitated aggregation of the Ag NPs. However, the particles still showed the typical absorption peak at $400 \mathrm{~nm}$, even in DMEM with $10 \% \mathrm{FBS}$ at $37^{\circ} \mathrm{C}$.

\subsection{Influence of pH on the stability of the Ag NPs}

In an earlier study, a large change in the $\mathrm{pH}$ of the solution greatly reduced the stability of the Ag NPs [25]. In the current study, we demonstrated that in a typical environmental and over the biological $\mathrm{pH}$ range of 4.2-8.4, changes in the $\mathrm{pH}$ did not alter the stability of the Ag NPs (Figure 7).

\subsection{Influence of environmental and biological factors on size and Zeta potential of Ag NPs}

A change in the particle size is another indicator of reduced stability of the $\mathrm{Ag}$ NPs. In $0.2 \mathrm{~mol} / \mathrm{L} \mathrm{CaCl}_{2}$ or $\mathrm{FBS}$-free DMEM, the particle size increased up to a microscale, especially at $37^{\circ} \mathrm{C}$. But in $0.2 \mathrm{~mol} / \mathrm{L} \mathrm{NaCl}$ and $\mathrm{DMEM}$ with $10 \%$ FBS, there were no large changes in the particle size (Figure 8). This observation is consistent with the results from UV-Vis spectroscopy (Figures 1, 2, 5 and 6).

The Zeta potential was measured to investigate the 

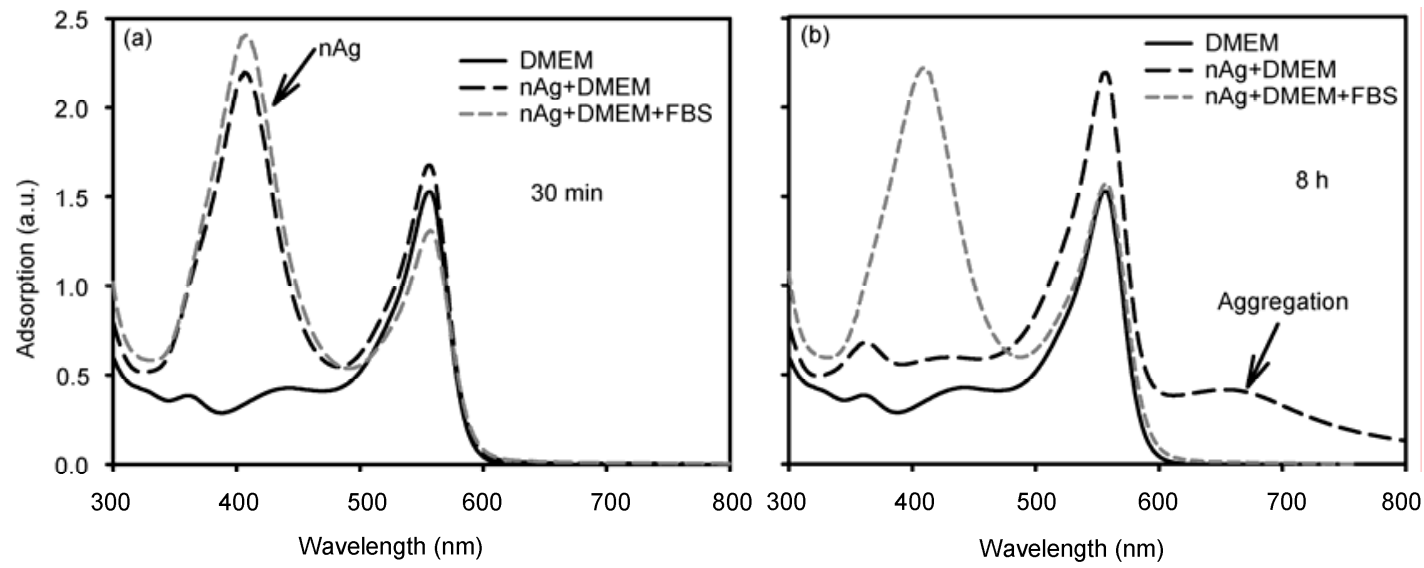

Figure 5 UV-Vis adsorption spectra of Ag NPs exposed to DMEM and DMEM+FBS. Peak at $550 \mathrm{~nm}$ is the typical absorption peak of phenol red.
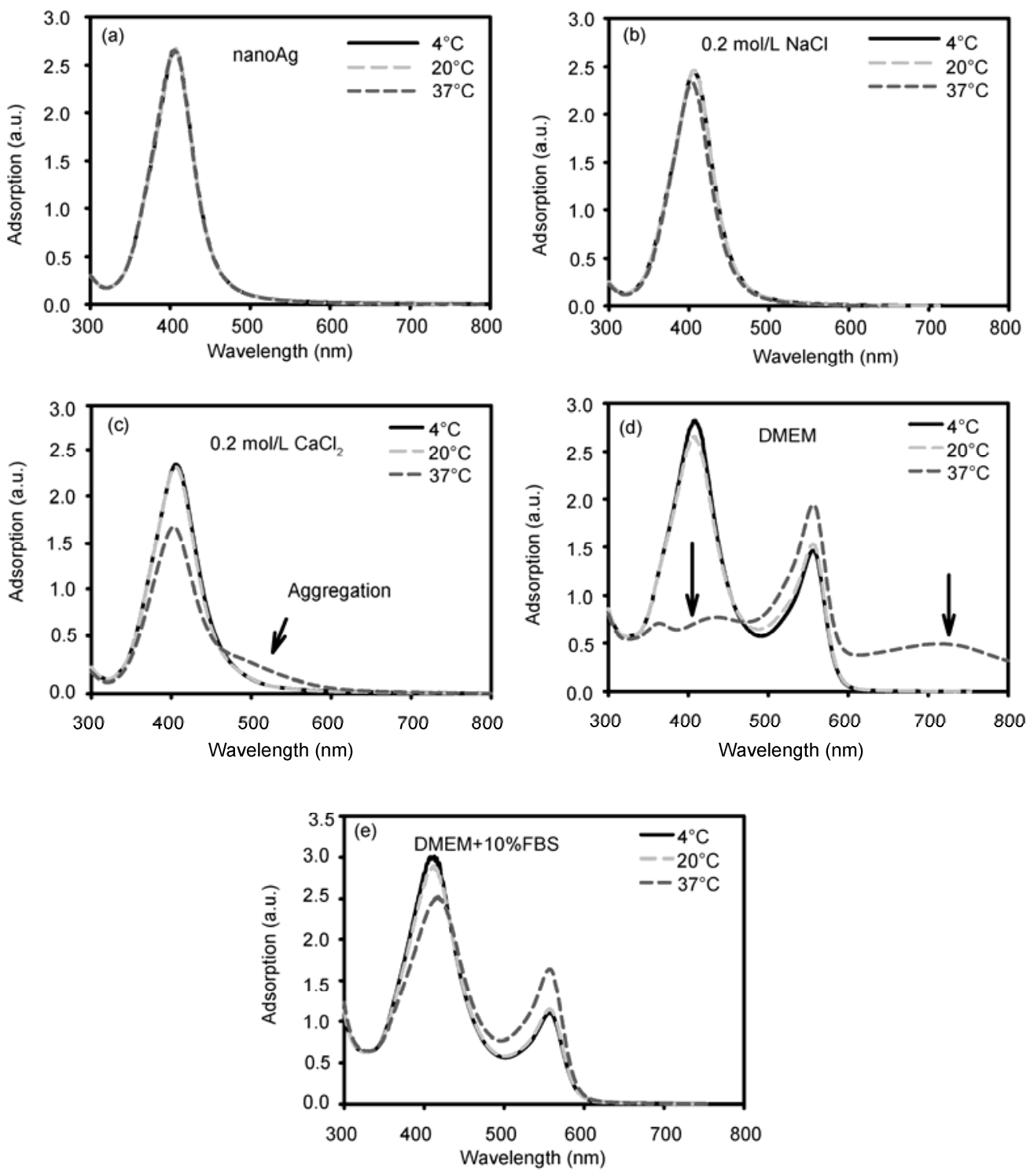

Figure 6 The UV-Vis adsorption spectra of Ag NPs suspended in various solutions at 4,20 and $37^{\circ} \mathrm{C}$. 


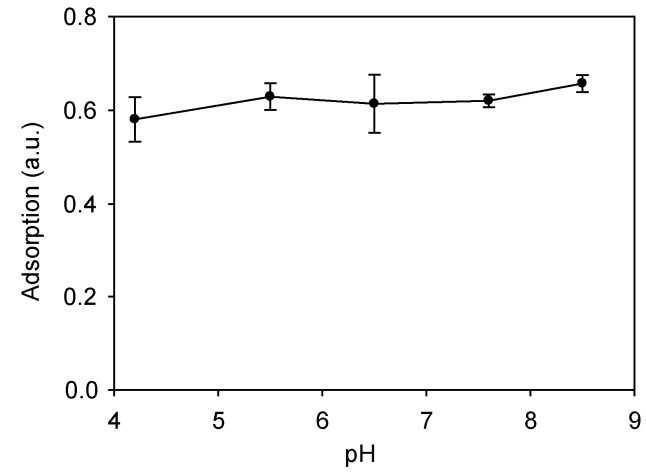

Figure 7 The absorbance of the Ag NPs at $400 \mathrm{~nm}$ from $\mathrm{pH}$ 4.2-8.4.

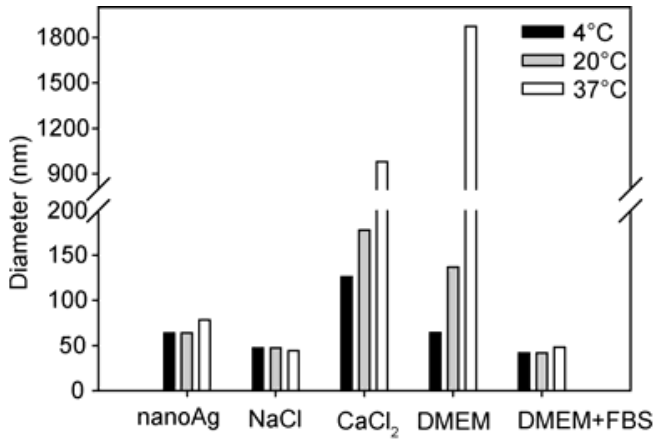

Figure 8 Influence of ionic strength, valence, temperature, and FBS on the size of Ag NPs.

influence of various solutions on the surface charge of the Ag NPs. In $0-0.5 \mathrm{~mol} / \mathrm{L} \mathrm{CaCl}_{2}$ or $\mathrm{NaCl}$ solution, the zeta potential of the Ag NPs increased from negative to positive (Figure 9). As biological media could not be assessed by this method, no data is presented for the zeta potential of the Ag NPs in DMEM and DMEM+FBS.

\subsection{Release of silver ions in various suspensions of $\mathrm{Ag}$ NPs}

The toxicity of Ag NPs arising from the release of free silver ions has been well documented [26]. The release of silver ions is also a reflection of the stability of the Ag NPs. In suspensions of $0-1 \mathrm{~mol} / \mathrm{L} \mathrm{CaCl}_{2}$ and $\mathrm{NaCl}$, DMEM or DMEM+FBS, the release of silver ions decreased from $3.0 \%$ to $0.5 \%$ (Figure 10), which suggests that the activity of the Ag NPs reduced. This might occur because of the aggregation or protein binding of the Ag NPs (Figure 11).

\section{Conclusion}

Ionic strength, valence, temperature, and some biological molecules were found to affect the stability of Ag NPs. The addition of DMEM and an increase in the ionic strength and valence all resulted in aggregation of the Ag NPs. The aggregation process was also promoted by an increase in the
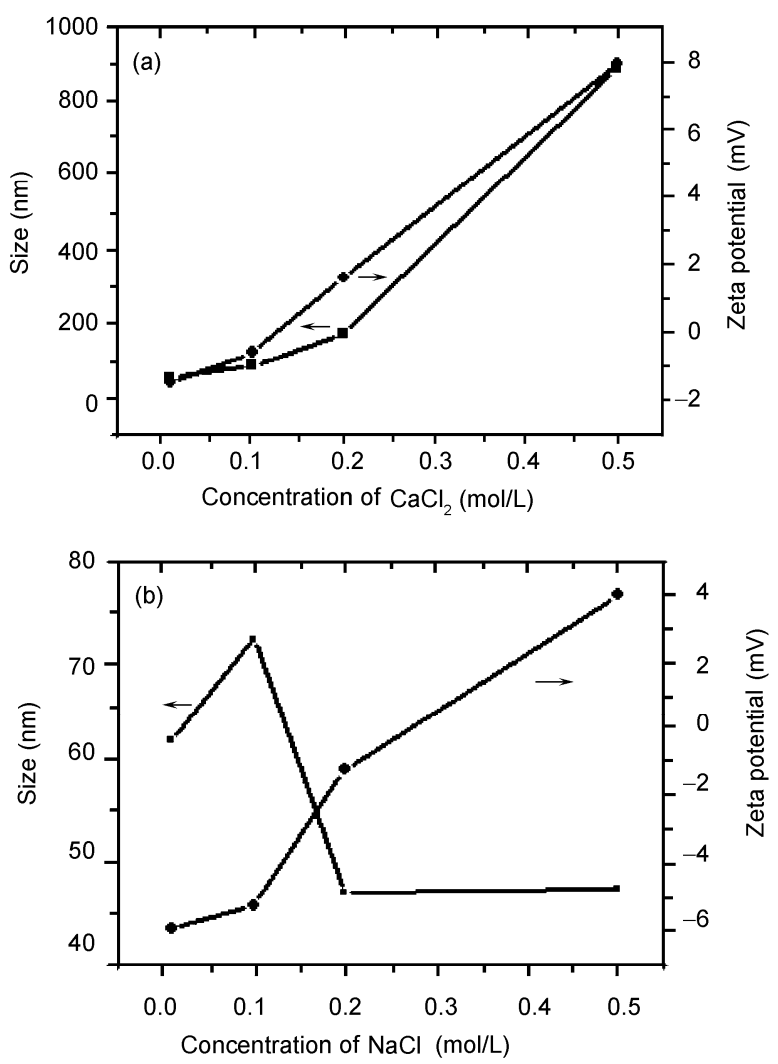

Figure 9 The Zeta potential of Ag NPs in $0-0.5 \mathrm{~mol} / \mathrm{L} \mathrm{CaCl}_{2}$ (a) and $\mathrm{NaCl}(b)$.

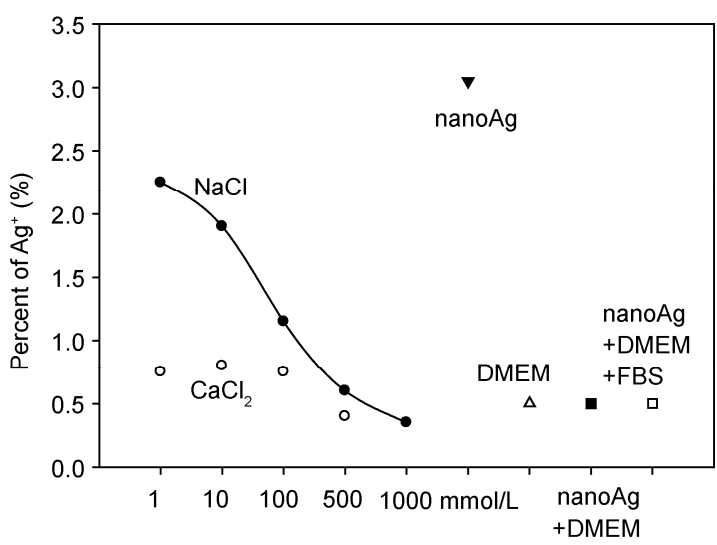

Figure 10 The release of silver ions in various suspensions of Ag NPs.

solution temperature. However, the presence of FBS in the cell culture medium helped maintain the stable dispersion of the Ag NPs. The stability of the Ag NPs was minimally affected by a change in $\mathrm{pH}$ within a range of 4.2-8.4. All tested solutions reduced the release of silver ions from the Ag NPs. These findings are summarized in Figure 11. The electrical double layer of the Ag NPs was not destroyed in weak ionic solutions or when the NPs were coated in proteins. This resulted in stable dispersions of the Ag NPs. By contrast, in stronger ionic solutions or those that lacked proteins, the electrical double layer collapsed and this led to 


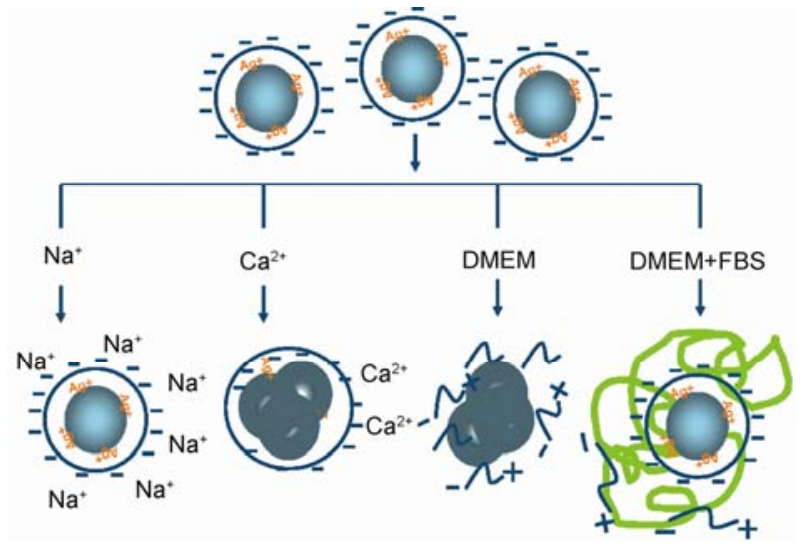

Figure 11 Summary of the influence of various factors on the stability of Ag NPs.

\section{aggregation of the Ag NPs.}

In the typical conditions of in vitro culture media, the Ag NPs could maintain their nanoscale features. Therefore, they should remain stable under in vivo conditions. These results will allow further investigation of the in vitro and in vivo toxicity of Ag NPs.

This work was supported by the National Basic Research Program of China (2009CB421605), the National Natural Science Foundation of China (B20977100), and the Knowledge Innovation Program of the Chinese Academy of Sciences (KZCX2-YW-420-21).

1 McNeil S E. Nanotechnology for the biologist. J Leukocyte Biol, 2005, 78: 585-594

2 Fu C C, Lee H Y, Chen K, et al. Characterization and application of single fluorescent nanodiamonds as cellular biomarkers. Proc Natl Acad Sci USA, 2007, 104: 727-732

3 Mann D, Kato Y K, Kinkhabwala A, et al. Electrically driven thermal light emission from individual single-walled carbon nanotubes. Nat Nanotechnol, 2007, 2: 33-38

4 Kim J H, Kim J S, Choi H, et al. Nanoparticle probes with surface enhanced raman spectroscopic tags for cellular cancer targeting. Anal Chem, 2006, 78: 6967-6973

5 Geho D H, Jones C D, Petricoin E F, et al. Nanoparticles: Potential biomarker harvesters. Curr Opin Chem Biol, 2006, 10: 56-61

6 Chen X, Schluesener H J. Nanosilver: A nanoproduct in medical application. Toxicol Lett, 2008, 176: 1-12

7 Yan W J, Wang R, Xu Z Q, et al. A novel, practical and green synthesis of ag nanoparticles catalyst and its application in threecomponent coupling of aldehyde, alkyne, and amine. J Mol Catal A-Chem, 2006, 255: 81-85

8 Deng J P, Shih W C, Mou C Y. Electron transfer-induced hydrogenation of anthracene catalyzed by gold and silver nanoparticles. J Phys
Chem C, 2007, 111: 9723-9728

$9 \mathrm{Yu} \mathrm{K} \mathrm{N,} \mathrm{Lee} \mathrm{S} \mathrm{M,} \mathrm{Han} \mathrm{J} \mathrm{Y,} \mathrm{et} \mathrm{al.} \mathrm{Multiplex} \mathrm{targeting,} \mathrm{tracking,} \mathrm{and}$ imaging of apoptosis by fluorescent surface enhanced raman spectroscopic dots. Bioconjugate Chem, 2007, 18: 1155-1162

10 Cho K H, Park J E, Osaka T, et al. The study of antimicrobial activity and preservative effects of nanosilver ingredient. Electrochim Acta, 2005, 51: 956-960

11 Rezaei-Zarchi S, Saboury A A, Norouzi P, et al. Use of silver nanoparticles as an electron transfer facilitator in electrochemical ligandbinding of haemoglobin. J Appl Electrochem, 2007, 37: 1021-1026

12 Blaser S A, Scheringer M, MacLeod M, et al. Estimation of cumulative aquatic exposure and risk due to silver: Contribution of nano- functionalized plastics and textiles. Sci Total Environ, 2008, 390: 396-409

13 Benn T M, Westerhoff P. Nanoparticle silver released into water from commercially available sock fabrics. Environ Sci Technol, 2008, 42: 4133-4139

14 Choi O, Hu Z Q. Size dependent and reactive oxygen species related nanosilver toxicity to nitrifying bacteria. Environ Sci Technol, 2008, 42: 4583-4588

15 Lu W T, Senapati D, Wang S G, et al. Effect of surface coating on the toxicity of silver nanomaterials on human skin keratinocytes. Chem Phys Lett 2010, 487: 92-96

16 Navarro E, Piccapietra F, Wagner B, et al. Toxicity of silver nanoparticles to chlamydomonas reinhardtii. Environ Sci Technol, 2008, 42: 8959-8964

17 Wang G, Zhang Y, Cui Y P, et al. Study on the behavior of hyper-rayleigh scattering for silver nanoparticles with aggregation effects. J Phys Chem B, 2005, 109: 1067-1071

18 Gilbert B, Ono R K, Ching K A, et al. The effects of nanoparticle aggregation processes on aggregate structure and metal uptake. J Colloid Interf Sci, 2009, 339: 285-295

19 Wick P, Manser P, Limbach L K, et al. The degree and kind of agglomeration affect carbon nanotube cytotoxicity. Toxicol Lett, 2007, 168: $121-131$

20 Carlson C, Hussain S M, Schrand A M, et al. Unique cellular interaction of silver nanoparticles: Size-dependent generation of reactive oxygen species. J Phys Chem B, 2008, 112: 13608-13619

21 Su X D, Kanjanawarut R. Control of metal nanoparticles aggregation and dispersion by pna and pna-DNA complexes, and its application for colorimetric DNA detection. ACS Nano, 2009, 3: 2751-2759

22 French R A, Jacobson A R, Kim B, et al. Influence of ionic strength, $\mathrm{ph}$, and cation valence on aggregation kinetics of titanium dioxide nanoparticles. Environ Sci Technol, 2009, 43:1354-1359

23 Kittler S, Greulich C, Gebauer J S, et al. The influence of proteins on the dispersability and cell-biological activity of silver nanoparticles. $\mathrm{J}$ Mater Sci, 2009, 20: 512-518

24 Greulich C, Kittler S, Epple M, et al. Studies on the biocompatibility and the interaction of silver nanoparticles with human mesenchymal stem cells (hmscs). Langenbeck Arch Surg, 2009, 394: 495-502

25 Sun L L, Wei G, Song Y H, et al. Controlled organization of silver nanoparticles into network assemblies by tuning ph values. Chem Lett, 2007, 36: 610-611

26 Johnston H J, Hutchison G, Christensen F M, et al. A review of the in vivo and in vitro toxicity of silver and gold particulates: Particle attributes and biological mechanisms responsible for the observed toxicity. Crit Rev Toxicol, 2010, 40: 328-346

Open Access This article is distributed under the terms of the Creative Commons Attribution License which permits any use, distribution, and reproduction in any medium, provided the original author(s) and source are credited. 